\title{
ZUR ARBEITSGESETZGEBUNG ALGERIENS
}

\author{
VON GUNTER WiedENSOHLER
}

Das am 5. August 1978 verabschiedete „Allgemeine Statut für Beschäftigte“ (statut général des travailleurs $)^{1}$ schafft für die unselbständige Erwerbstätigkeit in der Volksrepublik Algerien erstmals einen einheitlichen rechtlich-organisatorischen Rahmen. Die umfangreiche Kodifikation regelt die Beziehungen zwischen dem sozialistischen Unternehmen und dem Kollektiv der Arbeitenden und dessen Mitgliedern. In Gesetzesform gebracht sind unter anderem zwei Charakteristika des sozialistischen Arbeitsrechts: die Mitwirkung und Mitbestimmung des Werktätigen, die sich auch in der Beteiligung an den Wirtschaftsergebnissen des Unternehmens ausdrückt, und die Realität des Rechts auf Arbeit, das sich mittelbar etwa als Verbot der Zwangsarbeit und der Störung des Arbeitsfriedens konkretisiert, aber auch als Regelung der Berufsausbildung und der Arbeitsvermittlung 2 .

\section{Rechte der Arbeitnehmer}

Nach Vollendung seines 16. Lebensjahres ist jeder Staatsbürger im Einklang mit den Grundsätzen der Verfassung ${ }^{3}$ und der Nationalcharta ${ }^{4}$ berechtigt, eine seiner Vorbildung und Eignung entsprechende Berufstätigkeit auszuüben. Die Verwirklichung dieser Möglichkeit wird nach algerischem Selbstverständnis vor allem durch die ökonomischen und sozialen Grundgedanken des Sozialismus gewährleistet. Die gesetzliche Verankerung des ,,Rechts auf Arbeit" bedeutet daher keinen Anspruch auf Beschäftigung oder gar auf eine bestimmte Art von Tätigkeit, sondern lediglich die Verpflichtung des Staates zu einer konsequenten Politik der Vollbeschäftigung. Demgegenüber ist die Annahme eines subjektiven Rechts auf Arbeit zweifellos dann zu bejahen, wenn es um den Bestandsschutz des Arbeitsverhältnisses geht, also darum, die einmal erworbene berufliche Stellung zu halten ${ }^{5}$.

Das Gesetz verbietet jede willkürliche Ungleichbehandlung und gestattet Differenzierungen zwischen den Beschäftigten allein aufgrund ihrer Fähigkeiten und Leistungen. Ausdrücklich heißt es hierzu, daß die Arbeit - entgegen einer verbreiteten Praxis - bei gleicher persönlicher Eignung und gleichem persönlichen Einsatz nicht unterschiedlich vergütet werden darf. Gegenstand des rechtlichen Schutzes ist nunmehr auch die Würde des Arbeitnehmers, der vielfach unter der Willkür seiner Vorgesetzten zu leiden hat. Die Skala der entsprechenden Verhaltensweisen algerischer Arbeitgeber reicht von der offen zur Schau getragenen Geringschätzung bis hin zu Demütigungen verschiedener $\mathrm{Art}^{6}$. Das Gesetz entspricht daher einem besonderen Anliegen der werktätigen Bevölkerung, wenn es dem Arbeitnehmer garantiert, daß er ,,in Ausübung seiner Funktionen bzw. Erfüllung seiner Aufgaben gegen jede Form von Beleidigung, Drohung, Gewalt und Unterdrückung geschützt" werde.

\footnotetext{
1 Loi no 78-12 du 5 août 1978 relative a u statut général des travailleurs: Journal Officiel de la République Algérienne Nr. 32 vom 8 . 8. 1978, S. 532-543.

$2 \mathrm{Zu}$ anderen sozialistischen Staaten vgl. etwa A. Weltner, Fundamental Traits of Socialist Labour Law, With Special Regard to Hungarian Legislation. Budapest 1070, S. $42 \mathrm{ff}$., $97 \mathrm{ff}$.

3 Constitution de la République Algérienne Démocratique et Populaire, Artt. 44, 59: Journal Officiel Nr. 94 vom 24. 11. 1976 , S. 1049.

4 Ordonnance no 76-57 du 5 juillet 1976 portant publication de la charte nationale, in: Revue algérienne des sciences juridiques, économiques et politiques 19 (1976) Heft 3, S. $581 \mathrm{ff}$. Die Nationalcharta ist das politisch-ideologische Manifest Algeriens, das den wesentlichen Inhalt der geltenden Verfassung vorgezeichnet hat und als Maßstab für die Interpretation ihrer Vorschriften dient; dazu etwa B. Cubertafond, La constitution algérienne du 22 novembre 1976, in: Maghreb/Machrek 1977, Heft 75, S. $69,71$.

5 Siehe z. B. die Bestimmungen der Artt. $94 \mathrm{ff}$. des vorliegenden Gesetzes über den Schutz der Werktätigen gegen Massenentlassungen.

6 F. Ourabah, Quels sont les droits des travailleurs? in: El Moudjahid Nr. 4088 vom 17. 8. 1978.
} 
Eine Reihe weiterer Vorschriften bezweckt die Verbesserung des Unfallschutzes und - namentlich für berufstätige Frauen - der Hygiene am Arbeitsplatz. Der Arbeiter soll nicht länger in gesundheitsgefährdender Umgebung oder an schadhaften, ungesicherten Maschinen hantieren müssen und dadurch der erhöhten Gefahr von Unfällen und Berufskrankheiten ausgesetzt sein. Zugleich wird die Arbeitsmedizin wesentlich verbessert. Verschiedene soziale Einrichtungen, so etwa die Rehabilitationszentren, dienen der Erhaltung und Wiederherstellung der körperlichen und geistigen Leistungsfähigkeit.

$\mathrm{Zu}$ den politischen Rechten des Arbeitnehmers gehört vor allem das Recht auf regelmäßige Information durch die ihm vorgesetzten Instanzen in allen seine Berufstätigkeit betreffenden Fragen. Auf diese Weise will man nicht nur die Initiative und Kreativität des Arbeitnehmers wecken, sondern zugleich die Entscheidungsgremien von der Basis her kontrollieren. Während das Auskunftsrecht der Belegschaft in der Praxis des staatlichen Wirtschaftssektors durchweg Anerkennung und Beachtung gefunden hat ${ }^{7}$, sind die in Privatbetrieben tätigen Arbeitskräfte von diesem Ziel allerdings noch um einiges entfernt.

Der Gesetzgeber betont das Koalitionsrecht und erklärt den Eintritt der Berufstätigen in eine Gewerkschaft für wünschenswert. Die von den Arbeitnehmern gewählten Gewerkschaftsvertreter werden in der Ausübung ihrer Funktionen insofern geschützt, als sie während ihrer Amtsperiode weder gekündigt noch disziplinarisch zur Verantwortung gezogen werden können. Das Streikrecht wird bei privaten Beschäftigungsverhältnissen als eine Waffe zur Verteidigung legitimer Interessen für den Fall anerkannt, daß die Möglichkeiten einer einvernehmlichen Konfliktlösung erschöpft sind ${ }^{8}$. Im öffentlichen Wirtschaftssektor dagegen gelten Arbeitskampfmaßnahmen als illegal, weil man die Belange der Werktätigen durch ihre Beteiligung an der Unternehmensführung und am Ertrag hinreichend gewahrt sieht ${ }^{9}$. Aufgabe der staatlich gelenkten Union Générale des Travailleurs (UGTA) und ihrer Zweigorganisationen ist nicht die Führung von Arbeitskämpfen, sondern die ideologische Erziehung der Arbeiter und die Artikulierung gesellschaftspolitischer Forderungen ${ }^{\mathbf{1 0}}$.

\section{Pflichten der Arbeitnehmer}

Unabhängig von ihrem Rang in der Beschäftigungshierarchie sind die Erwerbstätigen gehalten, unter äußerstem Einsatz ihrer Fähigkeiten den Anforderungen ihrer Position zu genügen. Im Interesse des vorrangigen Zieles einer Erhöhung des Sozialprodukts geht es um die optimale Ausgestaltung der Arbeitsbedingungen. Den leitenden Kräften obliegt vor allem: die Durchsetzung der Prinzipien der sozialistischen Unternehmensverfassung ${ }^{\mathbf{1 1}}$, die Orientierung ihrer Maßnahmen an den Zielen des nationalen Entwicklungsplans, die Förderung von Arbeitsdisziplin und Verantwortungsbewußtsein sowie die Sorge um ein gutes Arbeitsklima im Kollektiv. Die Untergebenen haben die Anweisungen der zuständigen Funktionsträger nach bestem Können auszuführen, wobei folgende Grundsätze maßgebend sind: sinnvolle Ausnutzung der vorhandenen Kapazitäten; Steigerung der Qualität bei sinkenden

\footnotetext{
7 So vor allem seit Verkündung der sozialistischen Unternehmensverfassung - Charte de l'organisation socialiste des entreprises: Journal Officiel Nr. 101 vom 13. 12. 1971, S. $1346 \mathrm{ff}$.

8 Vgl. Ordonnance no 71-75 du 16 novembre 1971 relative aux rapports collectifs de travail dans le secteur privé: Journal Officiel Nr. 101 vom 13. 12. 1971 , S. 1354 f.

9 N. Grimaud, Organisation des entreprises socialistes algériennes, in: Maghreb 1972, Nr. 49, S. 48.

$10 \mathrm{Vgl}$. G. Wiedensohler, Betriebsverfassung und Mitbestimmung in Algerien, in: Verfassung und Recht in Ubersee 5(1972), Heft 3, S. $316 \mathrm{f}$.

11 Siehe Ordonnance no $71-74$ du 16 novembre 1971 relative à la gestion socialiste des entreprises: Journal Officiel Nr. 101 vom 13.12. 1971 , S. $1350 \mathrm{ff}$.
} 
Kosten; regelmäßige Beteiligung an den Aktionen der Arbeiterselbstverwaltung ${ }^{12}$; Kampf gegen jede Form von Lethargie, Parasitismus, Verschwendung und Bürokratie; Wahrung des Berufsgeheimnisses (vor allem bei bestehenden Auslandskontakten); allgemeine Festlegung auf die Grundideen der sozialistischen Arbeitsdoktrin.

Ferner trägt das Gesetz der Tatsache Rechnung, daß die in staatseigenen Unternehmen Beschäftigten ihre Stellung nicht selten zu ihrem eigenen finanziellen Vorteil mißbrauchen ${ }^{13}$. Aus diesem Grunde werden die Tätigkeit im staatlichen Wirtschaftssektor und die gleichzeitige Ausübung eines privaten Gewerbes - auch durch den Ehegatten - für miteinander unvereinbar erklärt. Die von einem breiten öffentlichen Konsens getragene Regelung bezweckt die Unterbindung eines persönlichen Profitstrebens, das allzu leicht mit den Interessen der Allgemeinheit kollidiert.

\section{Das Arbeitsverhältnis}

Einstellungen sind nur zulässig zur Schließung einer effektiv vorhandenen Beschäftigungslücke. Neu eingerichtete oder frei gewordene Stellen sind in erster Linie durch einen Rückgriff auf die Arbeitskraftreserven des jeweiligen Unternehmens zu besetzen. Ausländer dürfen nur eingestellt werden, soweit die Nachfrage auf dem inländischen Arbeitsmarkt nicht gedeckt werden kann. Die anerkannten Vorkämpfer der nationalen Unabhängigkeit (Moudjahidoun $)^{\mathbf{1 4}}$ wie auch die Witwen Gefallener werden vorrangig berücksichtigt. Für Körperbehinderte sind geeignete Tätigkeiten vorgesehen sowie verschiedene weitere Erleichterungen, etwa spezielle Lehrgänge und eigens eingerichtete Werkräume. Die Stellenbesetzung erfolgt aufgrund der wirtschaftlichen Bedarfslage nach Maßgabe der Vorbildung, Eignung und Neigung des Bewerbers. Soweit aus dienstlichen Gründen erforderlich, kann das beschäftigende Unternehmen dem Betreffenden eine andere Arbeit zuweisen, die seinen Fähigkeiten entspricht. Voraussetzung für die Zulässigkeit einer solchen Maßnahme ist jedoch unter allen Umständen, daß sie nicht zum Zwecke der Bestrafung geschieht und daß die finanzielle Lage des Arbeitnehmers sich nicht verschlechtert.

\section{Begründung des Arbeitsverhältnisses}

Die Einstellung ist nur statthaft, wenn der Kandidat durch eine ärztliche Untersuchung für gesundheitlich tauglich sowie durch Fachprüfungen und verschiedene Tests für beruflich geeignet befunden worden ist. Die Eingehung des individuellen Arbeitsverhältnisses erfolgt durch (nicht unbedingt schriftlichen) Vertrag, spätestens jedoch durch die tatsächliche Eingliederung des Bewerbers in den Arbeitsprozeß ${ }^{15}$. In der Regel wird das Arbeitsverhältnis auf unbestimmte Dauer begründet und beginnt mit einer Probezeit, die sechs bzw. bei Führungskräften neun Monate nicht überschreiten darf. Dem Stellenbewerber werden seine in der betreffenden Berufssparte früher abgeleisteten Dienstzeiten voll angerechnet. Vor Arbeitsantritt erhält er eine Einstellungsurkunde, in der seine Tarifeinstufung, Art und Umfang der zu leistenden Tätigkeit und die für ihn verbindlichen Rechtsvorschriften festgehalten sind.

12 Vgl. hierzu A. Tareb, Organisation des entreprises, Les travailleurs responsables àleur destin, in: El Djeich 10 (1972) Nr. 105, S. $11 \mathrm{ff}$. 13 F. Ourabah, Quels sont les obligations des travailleurs? in: El Moudjahid Nr. 4089 vom 18./19. 8. 1978.

$14 \mathrm{Im}$ Singular und mit dem bestimmten Artikel versehen ist dies der Titel einer führenden algerischen Tageszeitung, dieim vorliegenden Beitrag wiederholt zitiert wird.

15 Vgl. zu der entsprechenden Regelung in Ägypten G. Wiedensohler, Zum Entwurf eines neuen ägyptischen Arbeitsgesetzes, in: Orient 1970, Heft 1, S. 13. 
Untersagt ist die private Anwerbung, Auswahl und Einstellung von Arbeitskräften für Rechnung eines Dritten. Auf diese Weise wird das Arbeitsvermittlungsmonopol des Staates bekräftigt und zugleich ein Verbot der allgemein mißbilligten Arbeitnehmerüberlassungsverträge ausgesprochen ${ }^{\mathbf{1 6}}$. Im Interesse einer optimalen Nutzung des vorhandenen Arbeitskräftepotentials haben die Arbeitgeber eine Beschäftigungsbilanz zu erstellen, aus der sich ergibt: Zahl und Tätigkeit der gegenwärtig Beschäftigten, frei gewordene und neu geschaffene Stellen, Arbeitslage und Beschäftigungsmöglichkeiten des Unternehmens sowie die danach zu erwartende Zunahme oder Einsparung von Arbeitsplätzen.

Die Stetigkeit der algerischen Wirtschaftsentwicklung wird durch eine zunehmende Fluktuation der Arbeitskräfte gefährdet. So stößt die Realisierung der Planziele im Bildungswesen infolge der Abwanderung von Lehrkräften bereits auf ernste Schwierigkeiten. Das Gesetz schränkt deshalb die Mobilität der Beschäftigten ein, indem es die Zulässigkeit eines Stellenwechsels an die Voraussetzung knüpft, daß dem keine vorrangigen öffentlichen Interessen entgegenstehen. Wirksamer freilich würde der Staat - so eine verbreitete Meinung - die Unruhe auf dem Arbeitsmarkt unter Kontrolle bekommen, wenn er die zwischen verschiedenen Sektoren, Branchen und Berufsgruppen bestehenden Einkommensunterschiede beseitigte ${ }^{17}$.

\section{Inhalt des Arbeitsverhältnisses}

Die regelmäßige Arbeitszeit beträgt 44 Stunden pro Woche oder (bei monatlicher Vergütung) 190 Stunden im Monat ${ }^{18}$. Uberstunden sind nur ausnahmsweise statthaft, und zwar im Falle einer dringenden Notwendigkeit, eines wesentlichen Beitrages zur Verbesserung der Produktion sowie bei Vereinbarkeit mit den Erfordernissen der Vollbeschäftigungspolitik. Uber das Vorliegen dieser Voraussetzungen und den zeitlichen Umfang der zu leistenden Mehrarbeit entscheidet das Ministerium für Arbeit und Berufsausbildung von Fall zu Fall ${ }^{19}$. Die tägliche Arbeitszeit darf jedoch unter keinen Umständen 12 Stunden überschreiten und muß von angemessenen Pausen unterbrochen sein.

Erwerbstätige jedweder Position werden grundsätzlich nur für die Zeit entlohnt, in der sie ihrer Arbeitspflicht nachgekommen sind. Mit Rücksicht auf die zunehmende Praxis der Sammelbeförderung von Betriebsangehörigen gilt die Unpünktlichkeit durchweg als Arbeitsversäumnis. Höhe und Dauer der Lohnfortzahlung im Falle einer Arbeitsunfähigkeit durch Krankheit oder Unfall richten sich im wesentlichen nach den Vorschriften der Sozialgesetzgebung ${ }^{20}$. Ein völliger oder teilweiser Lohnausgleich wird außerdem gewährt bei Úbernahme eines Parteiamtes oder einer Gewerkschaftsfunktion, in der Zeit des gesetzlichen Mutterschaftsurlaubs, während der Teilnahme an offiziellen Sportveranstaltungen, desgleichen an Lehrgängen über politische und gesellschaftstheoretische Bildung, für die Dauer der obligatorischen Pilgerfahrt nach den heiligen Stätten des Islam etc.

Jedem Arbeitnehmer steht pro Woche ein Ruhetag zu und pro Kalenderjahr ein bezahlter Erholungsurlaub, dessen Dauer branchenweise unterschiedlich durch Dekret festgesetzt

16 So F. Ourabah, Les relations du travail, in: El Moudjahid Nr. 4090 vom 20. 8. 1978.

17 In diesem Sinne F. Ourabah, Les relations ... a. a. O. (vorangehende Fußnote).

18 So geregelt in der Arbeitszeitordnung, Ordonnance no 75-30 du 29 avril 1975 fixant la durée légale de travail: Journal Officiel Nr. 39 vom 16. 5. 1975, S. 430, in der Fassung der Ordonnance no 75-62 du 26 septembre 1975: Journal Officiel Nr. 80 vom 7. 10. 1975, S. 886.

19 Vgl. etwa arrêté du 8 juillet 1978 accordant à la société SNAM Progetti une dérogation exceptionnelle à la durée légale hebdomadaire de travail: Journal Officiel Nr. 35 vom 29. 8. 1978, S. 579.

20 Für den privaten Wirtschaftssektor vgl. auch die Artt. 236-240 der Ordonnance no 75-31 du 29 avril 1975 relative aux conditions générales de travail: Journal Officiel Nr. 39 vom 16. 5. 1975, S. $431 \mathrm{ff}$. 
wird. Der Urlaub darf nicht durch Geldleistungen abgegolten werden und ist nur in Ausnahmefällen auf das folgende Jahr übertragbar. Eine Betriebsordnung, zu deren Erlaß der Arbeitgeber verpflichtet ist, enthält allgemeine Verhaltensregeln für die Beschäftigten, Normen über die technische Organisation der Arbeit, Vorschriften zur Hygiene und Sicherheit am Arbeitsplatz wie auch einen Katalog von Zuwiderhandlungen nebst entsprechenden Sanktionen. Für den Fall eines drohenden oder bereits eingetretenen Arbeitskonflikts ist die Möglichkeit der Anrufung einer Schlichtungskommission und eines Schiedsgerichts vorgesehen.

\section{Auflösung des Arbeitsverhältnisses}

Als Beendigungsgründe nennt das Gesetz den Aufhebungsvertrag, den Ablauf des befristeten Arbeitsverhältnisses, die Aufgabe der Stellung, die disziplinarische Kündigung, den dauernden Verlust der Arbeitsfähigkeit, die Erreichung des Rentenalters sowie den Tod des Beschäftigten. Verschiedene Vorschriften sollen die Massenentlassung mit ihren weitreichenden sozialen Nachteilen erschweren. Zur Abwendung einer derartigen Maßnahme, die nur aus zwingenden betrieblichen Gründen erfolgen darf, ist der Arbeitgeber insbesondere verpflichtet, die Arbeitszeit zu reduzieren, Kurzarbeit einzuführen, die Altersgrenze vorzuverlegen und Beschäftigte in andere Unternehmensbereiche zu versetzen.

Einige afrikanische Arbeitsgesetzbücher, so das der Volksrepublik Kongo ${ }^{21}$, machen die Zulässigkeit einer Personaleinsparung davon abhängig, daß die fachliche Eignung, das Dienstalter und die familiären Verhältnisse der Erwerbstätigen angemessen berücksichtigt werden und daß der Betriebsrat rechtzeitig Gelegenheit zu einer Stellungnahme erhält ${ }^{22}$. Nach erfolgtem Personalabbau darf der Arbeitgeber in dem betreffenden Beschäftigungszweig bis auf weiteres weder Uberstunden arbeiten lassen noch Neueinstellungen vornehmen. Die durch eine Massenentlassung Betroffenen haben entsprechend ihren beruflichen Erfahrungen und Fähigkeiten einen Anspruch auf bevorzugte Wiedereinstellung.

\section{Das Arbeitsentgelt}

Die Prinzipien der Lohngestaltung und Lohnentwicklung sind für die unselbständig Tätigen in jedem Gesellschaftssystem von besonderer Bedeutung. Nach sozialistischer Doktrin obliegt die Lohnfestsetzung nicht den Partnern des Arbeits- oder des Tarifvertrages, sondern in der Regel allein dem Staat ${ }^{23}$. Allerdings haben die algerischen Werktätigen gemäß dem Grundsatz der kollektiven Unternehmensführung, die der jugoslawischen Arbeiterselbstverwaltung ${ }^{24}$ nachempfunden ist, innerhalb bestimmter Grenzen das Recht zur Mitwirkung bei der Verteilung des erzielten Gewinns. Maßstäbe für die Bemessung des Arbeitsentgelts sind die Erfordernisse der Entwicklung des Landes entsprechend den ökonomischen, sozialen und kulturellen Planzielen, die Daten über Produktion und Volkseinkommen, die Poli-

21 Loi no 45-75 du 15 mars 1975 instituant un Code du travail: Journal Officiel de la République Populaire du Congo(numéro spécial) vom 17. 3. 1975, Art. 39; dazu G. Wiedensohler, Grundlagen des Arbeitsrechts im frankophonen Afrika, in: Verfassung und Recht in Ubersee 11 (1978), Heft 3, S. 356 f.

22 Vgl. im einzelnen M. Kirsch, Le droit du travail africain, Bd. I, Paris 1975, S. 168-172.

23 Vgl. H.-J. Bartels, Das neue Arbeitsrecht der DDR, in: Rabels Zeitschrift für ausländisches und internationales Privatrecht 42 (1978), S. $537 \mathrm{f}$.

24 Dazu K. H. Jäger, Arbeiterselbstverwaltung und gesellschaftliches Eigentum/Ein Beitrag zum Status jugoslawischer Unternehmen, Stuttgart 1969, S. $107 \mathrm{ff}$ 
tik einer gerechten Einkommensverteilung sowie die zu erwartenden Auswirkungen auf das Wirtschaftswachstum. Das Arbeitsministerium setzt für jeden Tätigkeitsbereich Mindestlöhne fest ${ }^{25}$ und geht dabei von den allgemeinen Lebenshaltungskosten aus, deren Index jeweils in Gesetzesform veröffentlicht wird.

Die Reallöhne haben sich in den Jahren 1971 bis 1976 um durchschnittlich nur 5,6 \% erhöht, wobei freilich zu berücksichtigen ist, daß neben der Grundvergütung ein System von Zulagen, Prämien und verschiedenen Sozialleistungen besteht ${ }^{26}$. Auffallend ist eine zunehmende Differenzierung der Arbeitseinkommen nach Branchen und Qualifikationen. Hatte man die Lohnunterschiede zunächst generell durch eine Angleichung nach oben beseitigen wollen ${ }^{27}$, so läßt man das Mehrgehalt für qualifizierte Arbeitskräfte heute gern als Ausnahmeeinkommen erscheinen, das etwa für ,, unangenehme“ Tätigkeiten oder aufgrund der besonderen Gewinnsituation des Unternehmens gezahlt wird ${ }^{28}$. Der Gesetzgeber bekräftigt aufs neue das sozialistische Grundprinzip: , ,Jeder nach seinen Fähigkeiten, jeder nach seinen Leistungen“. Die Nivellierung sektoraler Lohnunterschiede ist indessen zu einem vorrangigen Planziel geworden. In diesem Sinne soll die Handhabung des geltenden Systems der Tarifeinstufung und Stellenbewertung künftig sorgfältiger kontrolliert werden und für die einzelnen Arbeitsbereiche eine Skala der Höchsteinkommen verbindlich sein. Eine von der Regierung eingesetzte Kommission - bestehend aus Mitgliedern der Einheitspartei sowie Repräsentanten der Gewerkschaften, der zuständigen Behörden und der Staatsunternehmen - soll Vorschläge zu Fragen der Lohngestaltung erarbeiten und die Durchführung der staatlichen Lohnpolitik fortlaufend überwachen.

\section{Lohnzahlung}

Der in Geld ausgedrückte Gegenwert für die geleistete Arbeit ist in regelmäßigen Abständen postnumerando zahlbar. Das beschäftigende Unternehmen hat den Lohn einschließlich aller Zulagen ordnungsgemäß zu berechnen und hierüber einwandfreie Unterlagen zu führen. Einbehaltungen vom Nettoverdienst sind nur in bestimmten Fällen (und jeweils in bestimmter Höhe) zulässig, so vor allem aufgrund eines Lohnpfändungsbeschlusses ${ }^{29}$. Einseitige Aufrechnungen des Unternehmens mit einem Anspruch gegen die Lohnforderung des Werktätigen sind daher ebensowenig statthaft wie die Abtretung der Lohnforderung an einen Dritten. Auch ist die rechtliche Situation des Arbeitnehmers dahin verbessert worden, daß seine Lohnansprüche nunmehr gegenüber Steuerforderungen und rückständigen Beiträgen zur Sozialversicherung Vorrang haben. Dem Kampf gegen die verbreitete Praxis der Ämterpatronage $\mathrm{e}^{\mathbf{3 0}}$ und der Korruption dient die nachdrückliche Feststellung, daß Gehaltszahlungen eines Unternehmens nur an die bei ihm effektiv Beschäftigten zulässig sind und daß ein Arbeitnehmer keinerlei Vorteile von (natürlichen oder juristischen) Personen annehmen darf, die Kontakte mit dem Arbeitgeber unterhalten.

\footnotetext{
25 So etwa durch Décret no 77-143 du 15 octobre 1977 portant fixation du salaire minimum garanti dans le secteur agricole: Journal Officiel Nr. 72 vom 16. 10. 1977, S. 866.

26 Siehe C. Pollak, Algerien vor dem neuen Vierjahresplan - Wirtschaftsstruktur und Entwicklungsprobleme, in: Internationales Afrikaforum 14 (1978), Heft 3, S. 266.

27 Révolution Africaine (Zentralorgan der algerischen Einheitspartei FLN) vom 26. 12. 1975, S. 11.

28 Dazu im einzelnen H. Elsenhans, Algerien - Koloniale und postkoloniale Reformpolitik, Hamburg 1977, S. $149-152$.

$29 \mathrm{Vgl}$. Ordonnance no $75-34$ du 29 avril 1975 relative á la saisie-arrêt et à la cession des rémunérations: Journal Officiel Nr. 39 vom 16.5. 1975, S. $461 \mathrm{f}$.

30 So R. N., La rémunération du travail, in: El Moudjahid Nr. 4091 vom 21. 8. 1978.
} 


\section{Lohnbestandteile}

Die Einstufung der Tätigkeit in den einzelnen Wirtschaftszweigen erfolgt anhand von Lohngruppenkatalogen, deren unterscheidende Merkmale im Verordnungswege geregelt werden. Úber die solcherart festgelegte Grundvergütung hinaus, die den üblichen Anforderungen der Einkommensstufe entspricht, erhält der Werktätige aus verschiedenen Gründen $\mathrm{Zu}$ schläge. Dies gilt z. B. für betriebsbedingte Arbeitserschwernisse - wie Schmutz, Lärm, Gefahr-, die nicht bereits durch Eingruppierung in die jeweilige Lohn- bzw. Gehaltsgruppe berücksichtigt sind. Schichtprämien werden an Beschäftigte gezahlt, die etwa aus Gründen einer besseren Auslastung der Produktionsanlagen eine ungünstigere Zeiteinteilung ihrer Arbeit hinnehmen müssen.

Wenn das Arbeitsergebnis des Werktätigen die für seine Vergütungsgruppe maßgebende Norm deutlich über- oder unterschreitet, werden innerhalb bestimmter Grenzen Leistungszuschläge gewährt bzw. Lohnkürzungen vorgenommen ${ }^{31}$. Durch eine innerbetriebliche Kommission ermittelt jedes Unternehmen regelmäßig anhand verschiedener Kriterien den Arbeitserfolg der Beschäftigten und bemißt danach die Höhe des variablen Lohnteils.

Neben der außergewöhnlichen Leistung ist der zulässige Mehraufwand an Arbeitszeit ein Grund für die Zahlung individueller Lohnzuschläge. Erfordert ein Arbeitsbereich häufig Uberstunden und ist dieser Umstand nicht in die Stellenbeschreibung aufgenommen, so kann die erhöhte Inanspruchnahme des Beschäftigten durch eine Pauschalentschädigung abgegolten werden. Voraussetzung hierfür ist jedoch, daß die solcherart aufgewerteten Stellen sowie Art und Umfang der Zusatzvergütung in einem Dekret fixiert werden und daß die Stellenbesetzung von der Aufsichtsbehörde genehmigt wird.

Die zunehmende Berufserfahrung des Arbeitnehmers wird in der Regel durch Beförderung, also Aufstieg in eine gehobene Position, honoriert. Ihr kann aber auch durch eine Dienstalterszulage Rechnung getragen werden, die erstmals nach dreijähriger Beschäftigung gezahlt wird und ihren Höchstbetrag - entsprechend der jeweiligen Tätigkeitsart - nach 25 bis 30 Jahren erreicht, wobei diese Zeitspanne weder ununterbrochen noch bei ein und demselben Arbeitgeber zurückgelegt werden muß. Die berufliche Erfahrung ergibt sich freilich nicht allein aus dem Dienstalter, sondern nicht zuletzt auch aus der (ebenfalls zu berücksichtigenden) fachlichen Weiterbildung des Arbeitnehmers. Schließlich erhält der Beschäftigte nach Maßgabe eines von Fall zu Fall erforderlichen Dekretes einen Gebietszuschlag für Arbeiten in entwicklungspolitisch besonders förderungswürdigen Gegenden, Tätigkeitsbereichen, Projekten und Wirtschaftszweigen. Im Verordnungswege wird festgelegt, daß die Summe der aufgeführten Verdienstzuschläge jeweils einen bestimmten Prozentsatz des Grundgehaltes nicht überschreiten darf ${ }^{32}$.

\section{Zusatzvergütung}

Diese Verbesserung des Arbeitseinkommens erscheint in der Form (a) eines Leistungsanreizes für die Tätigkeit im Kollektiv und (b) einer individuellen Beteiligung am Unternehmenserfolg.

a) Der Gegenwert für die Produktivität eines Kollektivs, das zur Erreichung eines bestimmten wirtschaftlichen Zieles eingesetzt ist, wird wie der Lohn monatlich entrichtet. Maßstäbe

31 Vgl. ,,Salaire et production“, in: El Moudjahid Nr. 4092 vom 22. 8. 1978

32 Vgl. R. N., La rémunération .... a. a. O. Fußnote 30. 
für die Bewertung des gemeinschaftlich herbeigeführten Arbeitsergebnisses sind: Umfang und Qualität der Produktion oder Dienstleistung, Zeitaufwand und Kosten sowie der Grad der Kapazitätsausnutzung. Die Höhe der auf den einzelnen Beschäftigten entfallenden $\mathrm{Zu}-$ satzvergütung (oder ihn treffenden Verdiensteinbuße) richtet sich nach seinem persönlichen Beitrag zum Arbeitserfolg des Kollektivs, bleibt aber im übrigen der näheren Bestimmung durch Regierungsdekret vorbehalten.

b) Daneben hat jeder Werktätige gemäß den Grundsätzen der Nationalcharta und der sozialistischen Unternehmensverfassung ${ }^{33}$ das Recht, aufgrund seines individuellen Einsatzes und unabhängig vom Tätigkeitsergebnis seiner Arbeitseinheit an der Verteilung der erwirtschafteten Mittel zu partizipieren ${ }^{\mathbf{3 4}}$. Soweit nicht für eine sinnvolle Investitionspolitik und Rücklagenbildung erforderlich, wird der Reinertrag des Unternehmens jährlich nach einem Modus unter die Beschäftigten verteilt, dessen Einzelheiten in jedem Wirtschaftszweig gesondert geregelt $\operatorname{sind}^{\mathbf{3 5}}$.

\section{Sozialleistungen}

Nicht minder bedeutend als die Vergütungsregeln sind die Maßnahmen zur Anhebung und Absicherung des sozialen Status der Werktätigen. Hierher gehört zunächst die Berufsausbildung mit ihrem günstigen Einfluß nicht nur auf die Verdienstmöglichkeiten, sondern auch auf die Qualität der Arbeit und die allgemeine Wirtschaftsentwicklung. Entwurf und Durchführung der Ausbildungsprogramme sind gemeinsame Aufgabe des Staates, der Unternehmen und der Gewerkschaften. Die Regierung oder der Arbeitgeber können eine Ausbildungsbeihilfe gewähren, wenn der Empfänger sich vertraglich verpflichtet, nach Abschluß seiner Lehre eine gewisse Zeit in dem Ausbildungsbetrieb tätig zu sein ${ }^{36}$.

Die den Beschäftigten und ihren Familien zur Verfügung gestellten Sozialeinrichtungen - für Gesundheit, Wohnung, Sport und Kultur - sollen das tägliche Leben erleichtern und das körperliche wie auch seelische Wohlbefinden erhöhen ${ }^{37}$. Um eine ungleichmäßige Verteilung dieser Vorteile zu vermeiden, ist eigens ein nationaler Ausgleichsfonds geschaffen worden. Die bisherigen Aufwendungen der Arbeitnehmer für Beförderung und Verpflegung sollen sich in dem Maße verringern (und schließlich ganz entfallen), wie der öffentliche Nahverkehr und das Kantinenwesen ausgebaut werden. Soweit der Werktätige von seinem Arbeitgeber untergebracht, verpflegt oder befördert wird, hat er sich freilich weiterhin an den entstehenden Kosten zu beteiligen. Nicht zuletzt ist eine Verbesserung der staatlichen Beihilfen für kinderreiche Arbeiterfamilien vorgesehen, wobei es dem Gesetzgeber insbesondere um das Wohl der Kinder geht.

Bei Arbeitsunfähigkeit infolge Krankheit oder Unfall, nach Eintritt in den Ruhestand wie auch im Falle des berufsbedingten Todes gewährt der Staat dem Beschäftigten bzw. seinen unterhaltsberechtigten Angehörigen ${ }^{38}$ eine den notwendigen Lebensunterhalt deckende Rente. Schwerbehinderte erhalten die Möglichkeit einer körperlichen und geistigen Rehabi-

33 Allgemein A. Tareb, Gestion socialiste des entreprises/la démocratie dans l'entreprise, in: El Djeich 1978, Nr. 186, S. 6-8.

34 Vgl. zum jugoslawischen Verteilungssystem H. Roggemann, Grundlagen des jugoslawischen Betriebsverfassungs- und Unternehmensrechts, Berlin 1968, S. $23 \mathrm{ff}$.

35 Näheres zur bisherigen Entwicklung bei S. Koulytchizky, L'autogestion, l'homme et l'état - l'expérience algérienne, Paris 1974, S. $359 \mathrm{ff}$.

36 R. N., La promotion et la protection sociale des travailleurs, in: El Moudjahid Nr. 4093 vom 23. 8. 1978.

37 Im einzelnen geregelt in Décrets nos 75/66-67 du 29 avril 1975 fixant les modalités de gestion des oeuvres sociales et la contribution des employeurs au financement: Journal Officiel Nr. 39 vom 16. 5. 1975, S. 465 f.

38 Zum Kreis der begünstigten Personen siehe Ordonnance no 74-87 du 17 septembre 1974 portant extension de la sécurité sociale: Journal Officiel Nr. 87 vom 27. 9. 1974, S. 826-828. 
litation mit dem Ziel der Wiederaufnahme ihrer früheren Tätigkeit; erforderlichenfalls werden sie für einen geeigneten anderen Beruf umgeschult. Die Höhe der Altersrente, die durch Pflichtbeiträge zur Sozialversicherung finanziert wird, bemißt sich nach dem früheren Gehalt und der Zahl der zurückgelegten Dienstjahre, darf jedoch unter keinen Umständen den gesetzlich garantierten Mindestlohn unterschreiten.

\section{Ausblick}

In den abschließenden Strafbestimmungen hebt das Gesetz noch einmal die Gesichtspunkte hervor, auf die es für die Verbesserung der Arbeitsbedingungen und die Erhöhung der Produktivität vor allem ankommt. Die Nichtbeachtung von Gesetzesvorschriften wie auch die Zuwiderhandlung gegen Tarifverträge und Betriebsordnungen werden in der Regel nur disziplinarisch geahndet, in schweren Fällen aber außerdem strafrechtlich verfolgt. So etwa der Verstoß gegen Bestimmungen des Arbeitsschutzes, die passive Bestechung, die Verletzung als solcher gekennzeichneter Betriebsgeheimnisse sowie jede Form der Störung des Arbeitsfriedens. Bisweilen bleibt es dem Staat oder dem beschäftigenden Unternehmen ausdrücklich vorbehalten, Schadenersatz von dem Deliquenten zu verlangen. Soweit es um die Frage der Ausübung zweier unvereinbarer Funktionen geht, hat jede Falschaussage oder Verschleierung der Tatsachen die frist- und entschädigungslose Auflösung des Arbeitsverhältnisses zur Folge ${ }^{39}$. Angesichts dieser entschiedenen Kampfansage gegen die vielbeklagten Grundübel der Nachlässigkeit, Rücksichtslosigkeit und Korruption ${ }^{\mathbf{4 0}}$ ist man in Algerien allgemein zuversichtlich, daß die Arbeitsbeziehungen fortan mehr denn je von Disziplin und Verantwortungsbewußtsein getragen werden ${ }^{\mathbf{4}}$.

$39 \mathrm{Vgl}$, „Ce qu'il faut retenir du statut général du travailleur“, in: El Moudjahid Nr. 4094 vom 24. 8. 1978

40 So etwa „Statut du travailleur/droits et devoirs, la juste mesure“, in: El Moudjahid Nr. 4095 vom 25./26. 8. 1978.

$41 \mathrm{Dazu}$ B. C. Nacer, Le statut général du travailleur/une assise juridique pour la bataille de la production, in: El Djeich 16 (1978), Nr. 184 , S. 6. 


\title{
Legalism Rampant: The Heritage of Imposed Law and the Constitution of Papua New Guinea.
}

\author{
By JOHN GOLDRING
}

The Constitution of Papua New Guinea, which came into force when Papua New Guinea became Independent, is autochthonous. It seeks to give, of its own force, legal effect to all the statute and judge-made law of the country, so that the authority of law is in no way dependent on any colonial law in force prior to Independence. It also seeks to establish the customs of the people, which were scarcaly recognised by the legal system prior to Independence, the status of ,underlying law' and to require the legislature and the courts to give effect to the traditions of the people. The Constitution itself calls for great significance to be attached to ,Papua New Guinean Ways”. The Constitution is the result of a process of popular consultation unparallelled in any other country.

Despite this, the Constitution of Papua New Guinea is firmly rooted in the traditions of Western culture, specifically, the Westminster system of government and the English Common Law. It is a complicated and highly legalistic document, which depends for its enforcement on judicial review and enforcement by a court and lawyers trained in the Common Law and brought up to respect the traditions of responsible and representative government. Despite the exhortations in the Constitution to respect and enforce the traditions of the country, it is itself an instrument which requires for its effectiveness a legal culture which is not indigenous to Papua New Guinea, but is, in fact, the heritage of the Common Law which was imposed upon the country by its colonial rulers.

\section{Labour Legislation in Algeria}

\section{By GUNTER WIEDENSOHLER}

The Algerian Law No. 78-12 of 5th August, 1978 (Statut général des travailleurs) is a significant example for the concept of labour law in a socialist country in development. A remarquably high importance is given to labour relations even in the Charter and the Constitution. Further it is surprising to see that freedom to strike does not exist in the public economic sector nor the principle of state sovereignty of collective bargaining, social security regulations. At various places there are hints to the social obligation to work, the principle of achievement motivated remuneration and to the significance of the vocational school system. The new law is expected to lead to a higher standard of discipline and responsibility within the labour relations.

\section{The Myth of the Hung-Kings and the National Self-Evidence of the Vietnamese}

The fact that marxism is the ruling ideology in Vietnam of ten seems to have the appearence to have overcome nationalism, which, otherwise, represents the most effective political ideology in the Third World. It is the task of this essay to prove by means of an analysis of the official interpretation on the myth of the Hung-kings that this fact is deceptive. These Hungkings were the legendary founders of the Vietnam state about whom there has been much report in the vietnamese annales. In the quest for nationalidentity the historians of the Socialist 